\title{
Inhibitory Effects of Litsea cubeba Oil and Its Active Components on Aspergillus flavus
}

\author{
Shiqi Xia $\mathbb{D}^{1},{ }^{1,2}$ Hong Lin $\left(\mathbb{D},{ }^{2}\right.$ Peilin Zhu $\mathbb{D}^{2}$, Peiling Wang $\mathbb{D}^{1},{ }^{1}$ Shengliang Liao $\left(\mathbb{D},{ }^{1}\right.$ \\ Shangxing Chen $\mathbb{D},{ }^{1}$ Zongde Wang $\mathbb{D},{ }^{1}$ and Guorong Fan $\mathbb{D}^{1}$ \\ ${ }^{1}$ College of Forestry, Jiangxi Agricultural University, \\ East China Woody Fragrance and Flavor Engineering Research Center of National Forestry and Grassland Administration, \\ Camphor Engineering Research Center of NFGA/Jiangxi Province, Nanchang 330045, China \\ ${ }^{2}$ Jiangxi Academy of Forestry, Nanchang 330032, China
}

Correspondence should be addressed to Zongde Wang; zongdewang@163.com

Received 26 August 2020; Revised 21 September 2020; Accepted 6 November 2020; Published 18 November 2020

Academic Editor: Kannan R. R. Rengasamy

Copyright ( $) 2020$ Shiqi Xia et al. This is an open access article distributed under the Creative Commons Attribution License, which permits unrestricted use, distribution, and reproduction in any medium, provided the original work is properly cited.

Aspergillus flavus (A. flavus) is a frequent harmful fungal pathogen. It can infect traditional Chinese medicine materials and release aflatoxin, to cause both economic and human health effects. By comparing the inhibitory potential of Litsea cubeba oil and its active components to A. flavus CGMCC 3.4408, citral was confirmed to be the main component that inhibits the growth of A. flavus CGMCC 3.4408, and the EC50 was $163.65 \mathrm{mg} \mathrm{L}^{-1}$. The inhibitory effect of citral on A. flavus CGMCC 3.4408 was studied for colony growth rate, mycelium biomass, aflatoxin production, and microstructure. Citral slowed down the growth rate of colonies and reduced mycelium biomass and toxin production. Moreover, citral altered the morphology of fungal spores and mycelium. In addition, citral also has the inhibitory effects on the isolates of $A$. flavus from moldy traditional Chinese medicinal materials. Thus, citral can be used as a potential agent to check the growth of A. flavus or related fungal strains.

\section{Introduction}

Aspergillus flavus (A. flavus or mildew) is a pathogenic fungus that exists widely in nature and its metabolite aflatoxin B1 (AFB1) is highly carcinogenic [1]. A. flavus easily infects huge number of important agricultural products, medicinal materials, and much more to count [2]. In China, traditional Chinese medicine prevails to large extent; hence the production and use of medicinal materials are quite frequent [3]. Some Chinese medicinal materials are easy to breed mildew due to poor storage environment and their own matrix conditions [4]. A. flavus is the dominant fungus that causes mildew of medicinal materials [5]. Moldy medicinal materials not only cause great economic losses, but also have plenty of food safety risks. Therefore, it is a dire need to identify certain novel ways to inhibit the growth of A. flavus in particular, which infects Chinese medicinal materials.
Multiple strategies have been designed to control fungal growth and aflatoxin production [6, 7]. However, there is a global trend on limiting chemical fungicides application in grains and food products due to their toxicity and persistence, as the fungicide residues migrate into the food chain, leading to serious health hazards for consumers [8,9]. Recently, many plant-based natural products which are safer compared to synthetic products have been considered as an alternative to these synthetic fungicides and preservatives [10-12]. Preventing A. flavus infections could be done by using plant essential oils, as they are classified as Generally Recognized As Safe (GRAS) and have low risk for developing resistance to pathogenic microorganisms [13]. Cymbopogon citratus essential oil, Zingiber officinale essential oil, and Satureja hortensis essential oil [14] are reported for significant reduction of $A$. flavus growth. 
Litsea cubeba (Lour.) Pers. is an endemic Chinese plant and is rich in phytochemicals [15]. It is mainly grown in different provinces of China (Hunan, Sichuan, and Jiangxi). A large amount of essential oils can be extracted from the fruits and leaves of Litsea cubeba [16]. Different studies showed that Litsea cubeba oil can effectively inhibit plant pathogenic fungi and bacteria $[17,18]$. It is a green and natural plant source as bacteriostatic agent. About $60-70 \%$ of Litsea cubeba oil comprised of citral, and researchers have focused on the bacteriostatic properties of citral $[19,20]$. However, other components such as citronellal, $\alpha$-terpineol, and linalool also have certain bacteriostatic properties but have not been studied well [21].

In the current study, sensitivity experiments and toxicity equations were designed to compare the inhibitory effects of Litsea cubeba oil, citral, citronellal, $\alpha$-terpineol, and linalool on A. flavus CGMCC 3.4408 qualitatively and quantitatively. Then, the effects of citral on the colony growth, the biomass, toxicity, and microstructure of A. flavus CGMCC 3.4408 shall be investigated systematically. In order to evaluate the potential of citral as a mildew preventive agent for Chinese medicinal materials, the strains of $A$. flavus shall be isolated and identified from moldy traditional Chinese medicinal materials. The virulence of different strains and inhibitory ability of citral shall be analyzed to lay a foundation for the follow-up studies.

\section{Materials and Methods}

2.1. Materials. A. flavus CGMCC 3.4408 was bought from China Center of Industrial Culture Collection. The strain was scratched on potato dextrose agar (PDA) medium and cultured at $26^{\circ} \mathrm{C}$ for a period of time.

Litsea cubeba oil and citral were bought from factory (Jiangxi Mashan Chemical Co., Ltd.). Citronellal, $\alpha$-terpineol, linalool, and other organic reagents were bought from Aladdin.

Moldy traditional Chinese medicinal materials used are Aurantii fructus and Alisma orientale, which were achieved from medicinal material base.

2.2. Preparation of A. flavus Spore Suspension. The culture dish of $A$. flavus that grew yellow-green spores was selected. About $5 \mathrm{~mL}$ sterile water (containing tween-80 with the volume ratio of $1 \%$ ) was taken in the dish. Then, the spores were scraped off gently with the inoculation ring. The sterile water containing spores was transferred to the centrifuge tube containing glass beads. The solution was shaken for $1 \mathrm{~min}$. Then, the impurities and hyphae were filtered out, and the filtrate was collected for use. The spores were counted using a blood cell counting plate and a microscope.

\subsection{Inhibitory Effect of Litsea cubeba Oil and Active Components on A. flavus 3.4408}

2.3.1. Determination of the Sensitivity. The bacteriostatic circle method was used in this study. The spore suspension was added to the sterilized medium, making the final concentration of spore suspension of $10^{5}-10^{6} \mathrm{CFU} \cdot \mathrm{mL}^{-1}$. Then, about $25 \mathrm{~mL}$ was poured to each medium dish. After medium solidification, a central hole $(6 \mathrm{~mm})$ was made. $30 \mu \mathrm{L}$ compounds whose concentrations were $150 \mathrm{mg} \mathrm{mL}^{-1}$, $225 \mathrm{mg} \mathrm{mL}^{-1}$, and $300 \mathrm{mg} \mathrm{mL}^{-1}$ were added to the hole, respectively, and cultivated under $26^{\circ} \mathrm{C}$ for $40 \mathrm{~h}$. Finally, bacteriostatic circle diameter was measured.

2.3.2. Inhibitory Effect of Litsea cubeba Oil and Active Components on Spores. Spore counting method was used in this study. Compounds of different concentrations were prepared. Compound $(1 \mathrm{~mL})$ was mixed with $24 \mathrm{~mL}$ medium and then poured into a flat plate, so that the final concentration of the compound would be $90 \mathrm{mg} \mathrm{L}^{-1}, 180 \mathrm{mg} \mathrm{L}^{-1}$, $360 \mathrm{mg} \mathrm{L}^{-1}, \quad 720 \mathrm{mg} \mathrm{L}^{-1}$, and $1440 \mathrm{mg} \mathrm{L}^{-1}$. $100 \mu \mathrm{L}$, $10^{6} \mathrm{CFU} \cdot \mathrm{mL}^{-1}$ spore suspension was uniformly coated on each dish. Then, the numbers of colonies were calculated after culture for $40 \mathrm{~h}$ at $26^{\circ} \mathrm{C}$. The experiment was repeated for three times.

\subsection{Effects of Citral on the Growth of A. flavus 3.4408 in Solid Medium}

2.4.1. Effects of Citral on the Growth Rate of Colony. Point contact method was used in this experiment. $1 \times 10^{5} \mathrm{CFU}$ spores were inoculated in the culture medium center and cultured at $26^{\circ} \mathrm{C}$ for $24 \mathrm{~h}$. Then, filter papers containing citral whose contents were $5 \mu \mathrm{L}$ and $10 \mu \mathrm{L}$ were pasted on the inner cover of the dish, respectively. The dishes were cultured at $26^{\circ} \mathrm{C}$ for 5 days. The colony diameter of A. flavus was measured every day. The linear equation between the colony diameter and time was analyzed. The slope was as the growth rate. The colony growth rate was calculated, and the influence of citral on the colony growth and morphology was analyzed.

2.4.2. Effects of Citral on A. flavus Microstructure. After 5 days of incubation in Section 2.4.1, $25 \mathrm{~mm}^{2}$ cubes were cut from the culture medium and then completely soaked in $5 \%$ glutaraldehyde solution. Then, through a series of pretreatments, the colony of $A$. flavus spores and hyphae were observed by using transmission electron microscope (SEM).

2.5. Effects of Citral on Growth and Toxicity of A. flavus 3.4408 in Liquid Medium. Five conical flasks containing $25 \mathrm{~mL}$ medium were added to spore suspension (the final concentration of spore suspension was $\left.10^{5} \mathrm{CFU} \cdot \mathrm{mL}^{-1}\right)$. Then, different concentration of citral was added to conical flask. The final concentrations of citral were $0 \mathrm{mg} \mathrm{L}^{-1}, 45 \mathrm{mg} \mathrm{L}^{-1}$, $180 \mathrm{mg} \mathrm{L}^{-1}, \quad 360 \mathrm{mg} \mathrm{L}^{-1}$, and $720 \mathrm{mg} \mathrm{L}^{-1}$, respectively. A. flavus was cultured at $26^{\circ} \mathrm{C}$ for 6 days. After culture, the hyphae were collected by suction filtration and weighed after desiccation. The filtrate was measured using aflatoxin $\mathrm{B}_{1}$ detection kit (MeiZheng Biotechnology Co., Ltd.). 


\subsection{Effects of Citral on A. flavus from Moldy Chinese Medicinal Materials}

2.6.1. Isolation and Identification of A. flavus from Moldy Chinese Medicinal Materials. The moldy Chinese medicinal materials were collected and eluted with sterile water. The eluted water points were placed on medium by spotting. Then, the dishes were cultured at $26^{\circ} \mathrm{C}$ for $1-2$ days. The single colonies were transferred to new dishes. After culture of single colonies for a period of time, mycelia were collected with sterilized dissecting blade and then crushed by grinding and added into solvent to extract DNA. The DNA of the suspected strain was sequenced by Tsingke Biological Technology Co., Ltd.

2.6.2. Determination of Toxicity of A. flavus from Chinese Medicinal Materials. Conical flask containing $25 \mathrm{~mL}$ medium was taken and added with spore suspension whose final concentration of spore suspension was $10^{5} \mathrm{CFU} \cdot \mathrm{mL}^{-1}$. The samples were incubated at $26^{\circ} \mathrm{C}$ for 6 days. After the culture period, the hyphae were collected by suction filtration and were weighed after desiccation. The content of AFB1 was detected using liquid chromatography.

2.6.3. Inhibitory Effect of Citral on A. flavus from Chinese Medicinal Materials. The bacteriostatic circle method and spore counting method were used (refer to the method of Section 2.3) to evaluate the inhibitory effect of citral on A. flavus that was isolated from moldy traditional Chinese medicine materials.

\subsection{Statistical Analysis}

2.7.1. Establishment of Toxicity Regression Equation. The experimental data in Section 2.3.2 was analyzed using DPS 7. 05 . In professional edition, the toxicity regression equation was established. Detailed method was that the concentration of the compounds was converted into logarithm and the inhibition rate was converted into probability. So the monadic linear regression relationship was developed between concentration and inhibition rate. Through the analysis of the significant linear relationship, the compounds can be compared to the strength of the pathogen virulence, and half effectively restrain mass concentration (EC 50) which was the important indicator of drug toxicity could be calculated.

\subsubsection{Sequence Analysis and Establishment of Phylogenetic} Trees. The resulting sequence in Section 2.6.1 was verified with Bioedit V7.0.9. The sequence was compared with NCBI, and the reliable sequence was selected and downloaded to establish the matrix. MEGA5.0 software was used for sequence alignment and pruning, and the first part was cut and saved for the construction of development trees. The phylogenetic tree was established using the maximum likelihood method using RaxML V7.2.6 software.
2.7.3. Significance Analysis. Significance analysis of the data was performed using one-way ANOVA (SPSS Statistics 21.0, SPSS Inc. Chicago, USA). Differences were considered significant when $P<0.05$.

\section{Results}

3.1. Screening of Highly Effective Inhibitory Components in Litsea cubeba Oil. The sensitivity of Litsea cubeba oil to A. flavus 3.4408 was compared with that of citral, citronellal, $\alpha$-terpineol, and linalool (Table 1). In general, the sensitivity of Litsea cubeba oil, citral, and citronellal was higher than that of $\alpha$-terpineol and linalool. When the concentration of the compounds was $150 \mathrm{mg} \mathrm{L}^{-1}$, Litsea cubeba oil and citronellal showed high sensitivity to A. flavus, while citral showed moderate sensitivity. At high concentration, the sensitivity of Litsea cubeba oil, citral, and citronellal was the same. The qualitative test showed that Litsea cubeba oil and its active components had inhibitory effect on A. flavus, but the inhibitory effect was different.

According to the results of spore counting method (Figure 1), Litsea cubeba oil and citral could completely inhibit the growth of A. flavus 3.4408 at high concentration. When the concentration of citral was $720 \mathrm{mg} \mathrm{L}^{-1}$, the inhibition rate of $A$. flavus reached $100 \%$, and the inhibitory effect was obviously better than that of $\alpha$-terpineol and linalool (inhibitory rate was less than 50\%). The establishment of toxicity equation is an effective method to quantitatively measure the inhibitory strength of active substances. The EC 50 is an important index to measure the antibacterial ability of compounds. Although there was no significant difference in the sensitivity of Litsea cubeba oil, citral, and citronellal to A. flavus, the EC 50 of Litsea cubeba oil and citral were significantly lower than that of citronellal (759.37 $\mathrm{mg} \mathrm{L}^{-1}$ ), which was $233.10 \mathrm{mg} \mathrm{L}^{-1}$ and $163.65 \mathrm{mg} \mathrm{L}^{-1}$, respectively (Table 2).

The results of sensitivity test and spore counting test showed that citral had the best inhibitory effect on A. flavus 3.4408 , and it was reported that the proportion of citral in Litsea cubeba oil was $60-70 \%$, which was the main antibacterial component in Litsea cubeba oil.

\subsection{Effects of Citral on the Growth of A. flavus 3.4408 in Solid} Medium. The change trend of the colony diameter of A. flavus 3.4408 along with the culture days in the whole culture process under the treatment of citral is shown in Figure 2. The bacterial colonies showed differences on the first day of culture. With the increase of culture time, the overall growth trend of bacterial colony diameter was slower, and the inhibition effect of citral on the growth of A. flavus was gradually enhanced. At the same culture time, the higher the citral concentration was, the smaller the colony diameter of A. flavus was. At the 5th day of culture, the colony diameters of A. flavus were $82.27 \%$ and $72.72 \%$ compared to the control group under the citral concentrations of $5 \mu \mathrm{L}$ and $10 \mu \mathrm{L}$, respectively. A linear equation (on the right side of Figure 2) with the slope of growth rate was obtained by plotting colony diameter against time. The growth rate of 
TABLE 1: The sensitivity of Litsea cubeba oil and its active components.

\begin{tabular}{lccc}
\hline Components & & Concentration $\left(\mathrm{mg} \cdot \mathrm{L}^{-1}\right)$ & \\
& 150 & 225 & 300 \\
\hline Litsea cubeba oil & $16.03 \pm 0.71 \mathrm{~mm}^{\mathrm{c} * *}$ & $20.10 \pm 0.78 \mathrm{~mm}^{\mathrm{a} * * * *}$ & $26.93 \pm 0.55 \mathrm{~mm}^{\mathrm{a} * * * *}$ \\
Citral & $14.83 \pm 0.25 \mathrm{~mm}^{\mathrm{c} *}$ & $26.93 \pm 0.45 \mathrm{~mm}^{\mathrm{b} * * * *}$ & $30.67 \pm 0.42 \mathrm{~mm}^{\mathrm{b} * * * *}$ \\
Citronellal & $16.17 \pm 1.26 \mathrm{~mm}^{\mathrm{a} * *}$ & $19.13 \pm 0.42 \mathrm{~mm}^{\mathrm{c} * * * *}$ & $28.83 \pm 1.04 \mathrm{~mm}^{\mathrm{c} * * * *}$ \\
$\alpha$-Terpineol & $12.47 \pm 0.50 \mathrm{~mm}^{\mathrm{b} *}$ & $17.00 \pm 0.50 \mathrm{~mm}^{\mathrm{d} *}$ & $18.63 \pm 0.40 \mathrm{~mm}^{\mathrm{d} *}$ \\
Linalool & $9.23 \pm 0.25 \mathrm{~mm}^{\mathrm{c} *}$ & $15.3 \pm 0.36 \mathrm{~mm}^{\mathrm{e} * *}$ & $17.07 \pm 0.31 \mathrm{~mm}^{\mathrm{e} * *}$ \\
\hline
\end{tabular}

Inhibitory zone diameter $>20 \mathrm{~mm}$, extreme sensitivity****; inhibitory zone diameter $15-20 \mathrm{~mm}$, high sensitivity ${ }^{* *}$; inhibitory zone diameter $10-15 \mathrm{~mm}$, moderate sensitivity*; inhibitory zone diameter $<10 \mathrm{~mm}$, low sensitivity ${ }^{*}$. Capital letters in each column showed significant differences $(P<0.05)$.

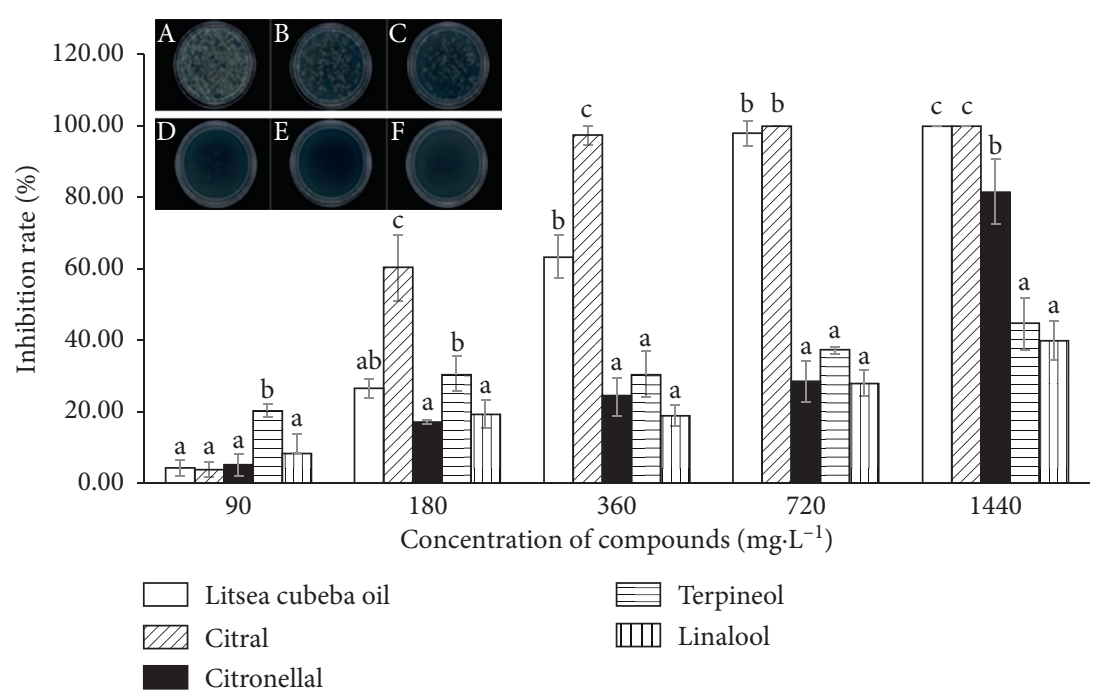

Figure 1: Spore counting method of Litsea cubeba oil and active components. $*$ A: control (untreated); B: citral at $90 \mathrm{mg} \mathrm{L} \mathrm{L}^{-1}$; C: citral at $180 \mathrm{mg} \mathrm{L}^{-1}$; D: citral at $360 \mathrm{mg} \mathrm{L}^{-1}$; E: citral at $720 \mathrm{mg} \cdot \mathrm{L}^{-1}$; F: citral at $1440 \mathrm{mg} \mathrm{L}^{-1}$. $* \mathrm{a}, \mathrm{b}$, and c show significant differences $(P<0.05)$.

TABLE 2: The toxicity equation of Litsea cubeba oil and active components.

\begin{tabular}{lccc}
\hline Components & Toxicity equation & Correlation coefficient $R^{2}$ & EC $50\left(\mathrm{mg} \cdot \mathrm{L}^{-1}\right)$ \\
\hline Litsea cubeba oil & $y=5.3493 x-7.6646$ & 0.9704 & 233.10 \\
Citral & $y=6.0740 x-8.4473$ & 0.9741 & 163.65 \\
Citronellal & $y=1.7972 x-0.1768$ & 0.9278 & 759.37 \\
$\alpha$-Terpineol & $y=0.5205 x+3.2106$ & 0.9655 & 2741.43 \\
Linalool & $y=0.8362 x+2.0715$ & 0.9636 & 3178.62 \\
\hline
\end{tabular}

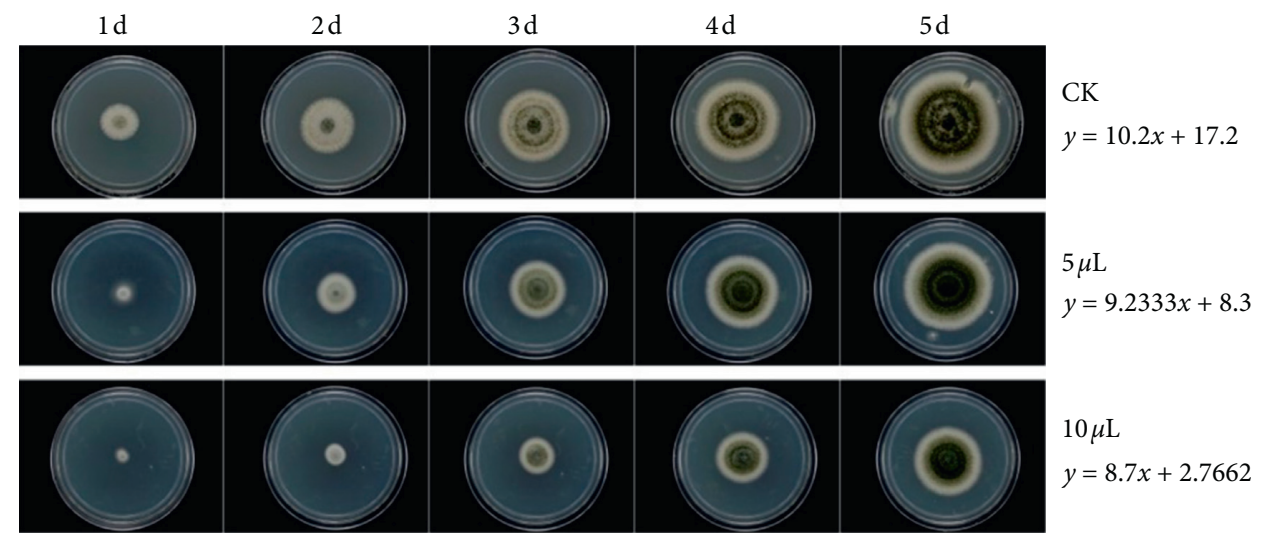

Figure 2: Effects of citral on colony growth of A. flavus 3.4408. 
A. flavus decreased with the increase of citral concentration. When the concentration of citral was $10 \mu \mathrm{L}$, the colony growth rate was reduced by $15 \%$. By observing the overall morphology of the colony, it was found that the addition of high concentration of citral could significantly reduce the diameter and growth of the colony, the spores in the colony were sparse, the spore production ability was decreased, and the physiological morphology was abnormal.

3.3. Inhibitory Effect of Citral on Growth and Toxicity of A. flavus 3.4408. The trend of growth and toxin production of A. flavus 3.4408 in the whole culture process with the change of culture days under the treatment with citral is shown in Figure 3. With the increase of the concentration of citral, the dry weight of A. flavus decreased, and the production of AFB1 also decreased. The concentration of citral was negatively correlated with the growth and toxin production. When the concentration of citral was $180 \mathrm{mg} \mathrm{L}^{-1}$, the dry weight of mycelia was only $0.004 \mathrm{~g}$, the inhibition rate of the growth was $98.79 \%$, and the inhibition rate of toxin production was more than $99 \%$. It can be concluded that citral can significantly inhibit the mycelium growth and the production of AFB1. According to the observation of mycelium pellet morphology of A. flavus (Figure 3), it can be found that large number of mycelia wound into pellets at low concentration, while, compared with the control group, mycelium pellets are smaller. When the concentration of citral reached $180 \mathrm{mg} \mathrm{L}^{-1}$, the mycelia sparsely formed into strips. The results showed that citral had a significant inhibitory effect on mycelium biomass synthesis and also had a strong inhibitory effect on mycelium morphology. Citral can inhibit the biosynthesis of the secondary metabolite AFB1 by inhibiting the growth of $A$. flavus. The inhibition of citral on the growth and toxin metabolism of A. flavus had double effects, and there was an obvious concentration-effect relationship.

3.4. Effect of Citral on the Structure of A. flavus 3.4408. In this study, scanning electron microscopy (SEM) was used to investigate the effects of different concentration of citral on the structure of A. flavus 3.4408, reflecting the degree of inhibitory effect of citral on the growth of A. flavus 3.4408 (Figure 4). The spores of the control group with no treatment were in full shape and in large number. The spores of the experimental group had pores on the surface and collapsed at the concentration of $5 \mu \mathrm{L}$, and the spore size was smaller than that of the control group. With the increase of the concentration of citral, the destruction of spore structure was more serious - the spore head burst and the spore shrank. When no citral treated, the hypha surface was smooth and the thickness was even. Under the action of citral, mycelia were disorderly arranged, tightly wound, and twisted. Therefore, it can be speculated that citral will destroy the germination and growth of spores, rupture the cell membrane, cause collapse and necrosis of mycelium, and thus affect the normal physiological metabolism of $A$. flavus.
3.5. Identification and Toxicity Determination of A. flavus from Traditional Chinese Medicine. Herein, two strains were isolated from moldy Chinese medicinal materials. DNA of these two strains was extracted and sequenced, and the sequencing results were compared in GenBank. Then, the sequencing results were analyzed by RaxML V7.2.6 software, and the maximum likelihood (ML) phylogenetic tree is shown in Figure 5. The phylogenetic tree consists of six species with a total of 19 representative strains and an outgroup. The sequences obtained in this study were clustered in the same branch as A. flavus, and the support rate reached $100 \%$. It was indicated that the isolated strains belong to A. flavus, named as ZK and ZX.

The toxicities test showed that there were significant individual differences among different strains of the same species, and the toxicities between A. flavus 3.4408 and ZK differed by three orders of magnitude. According to Chinese relevant provisions, the limit standard of AFB1 in Chinese medicinal materials is $5 \mu \mathrm{g} \mathrm{kg}^{-1}$. In present study, the toxic amount of $\mathrm{ZK}$ is $3.01 \mu \mathrm{g} \mathrm{kg}^{-1}$, with weak toxic capacity, while the toxic amount of $Z X$ is $345 \mu \mathrm{g} \mathrm{kg}^{-1}$. It was indicated that the A. flavus in moldy Chinese medicinal materials has certain toxic capacity, and its harm cannot be ignored.

3.6. Inhibitory Effect of Citral on ZK and ZX. According to the sensitivity test and spore counting test, the inhibitory effect of citral on ZK and ZX was researched. The sensitivity of citral on ZK and ZX was moderate (Figure 6). The inhibiting ability of citral on ZK was slightly stronger than ZX (Table 3). In high concentration, citral can completely inhibit the growth of ZK. The EC50 of ZK and ZX were $270.87 \mathrm{mg} \mathrm{L}^{-1}$ and $363.27 \mathrm{mg} \mathrm{L}^{-1}$, respectively. Combined with the experiment of strain 3.4408, it was concluded that the inhibitory effect of citral on the A. flavus with different sources was different and citral can be used as an effective bacteriostatic material to inhibit A. flavus from Chinese medicinal materials.

\section{Discussion}

In recent years, consumers demand for effective and safe natural products to control food spoilage without chemical residues has increased to great extent. Essential oils are aromatic and volatile, produced as secondary metabolites of plants. Most essential oils play an important role in reducing food spoilage. In general, terpenoids are the main constituents of most essential oils, including monoterpenes, sesquiterpenes, and their oxygenated derivatives. Among them, monoterpenes are the active antimicrobial compounds of essential oils. Citral is one of the most important representatives of open chain monoterpenes accounting for $60-70 \%$ of the total Litsea cubeba oil. Besides, citronellal, $\alpha$-terpineol, and linalool were accounted for the inhibition though their percentage in Litsea cubeba oil is low.

The sensitivity test can directly judge whether the compound is sensitive to the target bacteria, a way to screen sensitive compounds, and usually used as a preliminary study to find the inhibitory effects of essential oils on target 


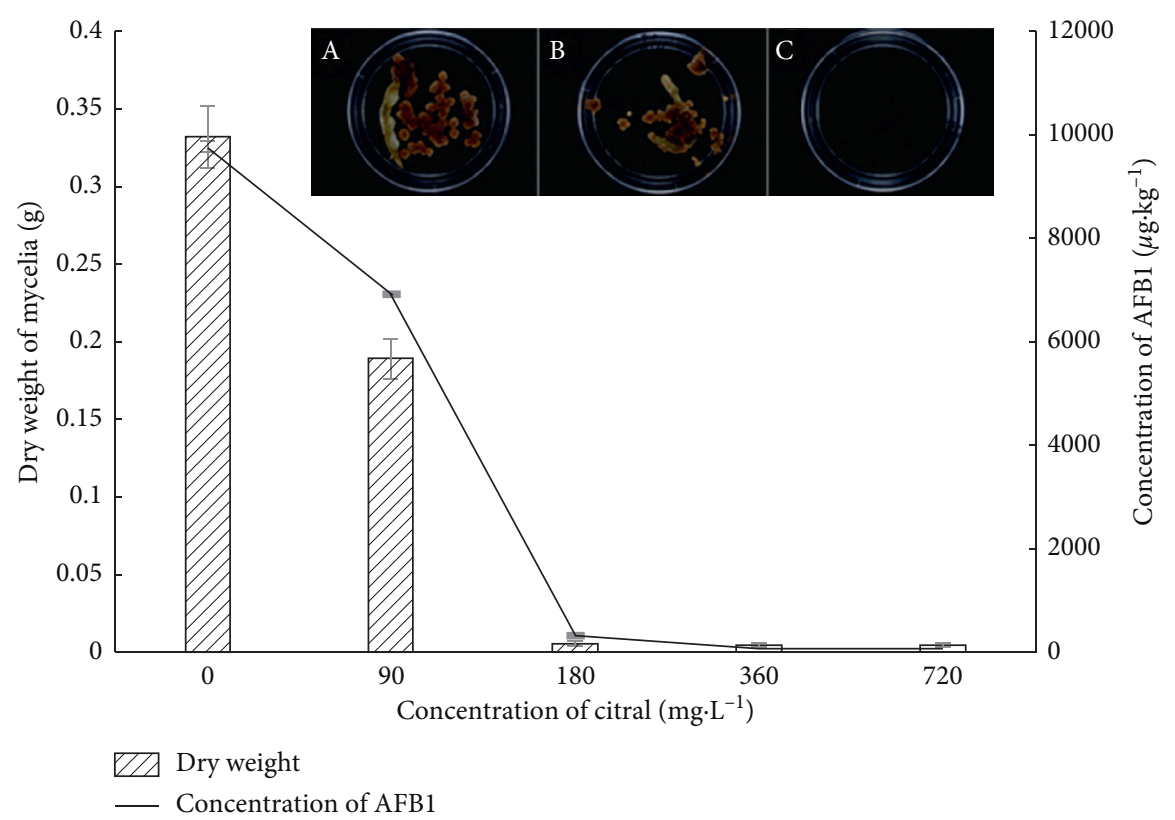

Figure 3: Inhibitory effect of citral on growth and toxicity of A. flavus 3.4408. *A: control (untreated); B: citral at $90 \mathrm{mg} \mathrm{L}^{-1}$; C: citral at $180 \mathrm{mg} \mathrm{L}^{-1}$.

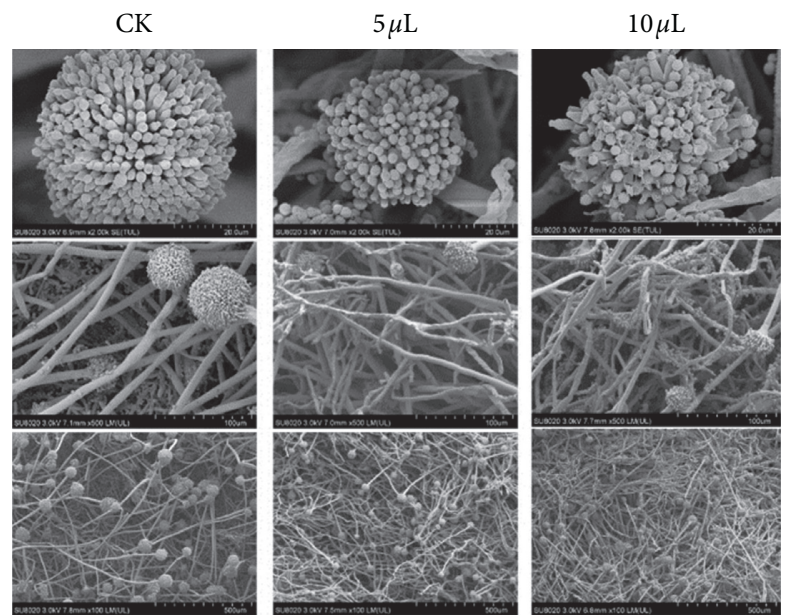

FIgURE 4: SEM of A. flavus 3.4408.

bacteria. However, it is necessary to calculate the half inhibition concentration or minimum inhibitory concentration in order to accurately judge the antibacterial ability potential of that particular compound. For instance, a study combined inhibition zone diameter with minimal inhibitory concentration to find antimicrobial activity of essential oils in the methanolic extracts of Thymus nummularius (Anzer tea) [22]. Therefore, these two methods were adopted to screen the compounds having strongest inhibitory ability for A. flavus 3.4408 for systematic bacteriostatic research and subsequent utilization. Herein, citral showed that it had best inhibition in the composition of Litsea cubeba oil.

Under both solid and liquid culture conditions, citral showed marked inhibition of A. flavus 3.4408. The citral mediated inhibition of $A$. flavus showed decline in growth in a time dependent fashion. Compared with the control, citral retarded the growth of hysteresis colony in the experimental group. Furthermore, citral had a concentration effect on the inhibition of $A$. flavus; i.e., the higher the concentration of citral, the smaller the colony diameter and the less bacterial biomass and toxin production are examined. Mycelial growth of $A$. flavus was reduced significantly at $150 \mathrm{mg} \mathrm{L}^{-1}$ concentration of Zingiber officinale essential oil [23], while $650 \mathrm{~g} \mathrm{~mL}^{-1}$ concentration of Cinnamomum zeylanicum essential oil showed MIC [24]. In the current study, the EC50 of citral on fungal spores was $163.65 \mathrm{mg} \mathrm{L}^{-1}$, and $180 \mathrm{mg} \mathrm{L}^{-1}$ concentration significantly inhibited the production of mycelia and toxins as comparable to the inhibition mediated by citral for A. flavus.

The results also showed that citral can potentially inhibit dry mycelium weight and the AFB1 synthesis of A. flavus 3.4408. Literature showed a direct correlation between 


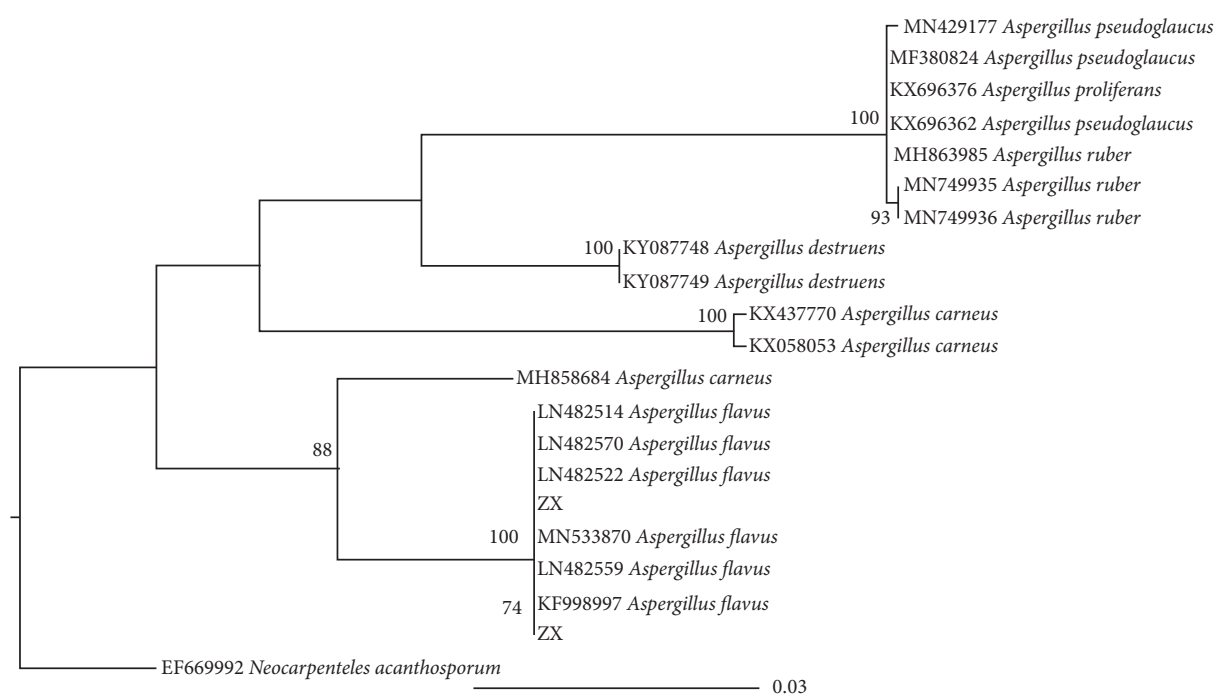

FIGURe 5: Phylogenetic tree shows the origin of two strains (ZK and ZX) of the fungus.
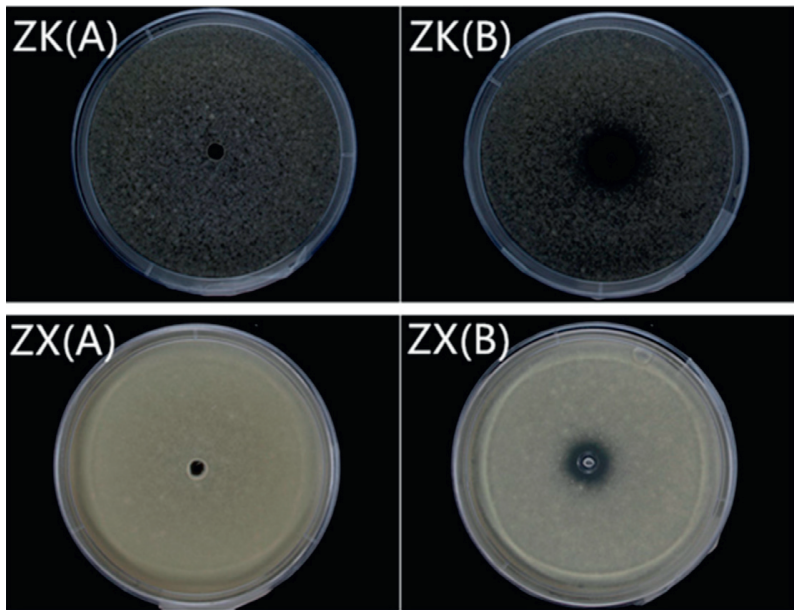

FIgURE 6: The sensitivity of citral on ZK and ZX. A: control (untreated); B: the experimental group $\left(150 \mathrm{mg} \mathrm{L}^{-1}\right)$.

TABLe 3: The toxicity equation of citral on ZK and ZX.

\begin{tabular}{|c|c|c|c|c|c|c|c|}
\hline \multicolumn{6}{|c|}{ Concentration $\left(\mathrm{mg} \cdot \mathrm{L}^{-1}\right)$} & \multirow{2}{*}{ Toxicity equation } & \multirow{2}{*}{$\operatorname{EC50}\left(\mathrm{mg} \mathrm{L}^{-1}\right)$} \\
\hline & 90 & 180 & 360 & 720 & 1440 & & \\
\hline $\mathrm{ZK}$ & $13.89 \% \pm 7.0 \%$ & $16.29 \% \pm 3.89 \%$ & $30.87 \% \pm 10.11 \%$ & $63.12 \% \pm 10.22 \%$ & $100 \%$ & $y=4.4807 x-5.9004$ & 270.87 \\
\hline ZX & $24.94 \% \pm 9.33 \%$ & $32.57 \% \pm 9.17 \%$ & $52.42 \% \pm 2.68 \%$ & $58.52 \% \pm 6.84 \%$ & $79.64 \pm 2.56 \%$ & $y=1.2217 x+1.8721$ & 363.27 \\
\hline
\end{tabular}

fungal growth and AFB1 production $[25,26]$. However, the inhibition of AFB1 production cannot be completely attributed to the insufficient fungal growth, but instead it can also be linked with the change in microstructure with following citral treatment. It is also elucidated that, citral combined with aflatoxin is a good strategy for complete removal of the fungus. Hence, determining the AFB1 suppression mechanism requires further investigation.

Most of the studies used type strains to carry out scientific research, while the current study explored the potential of citral as a mildew preventive agent for medicinal materials. It is not convincing to use only the type strains as the research object, but various strains of A. flavus isolated from moldy Chinese medicinal materials could be ideal strains. Based on the results, citral appeared to inhibit A. flavus from moldy Chinese medicinal materials.

\section{Conclusions}

The inhibitory effect of Litsea cubeba oil and its active components on A. flavus 3.4408 showed that citral had the best inhibitory effect on growth. The inhibition of bacterial growth rate, biomass, and AFB1 synthesis showed good bacteriostatic ability of citral to A. flavus strains (ZK and ZX) isolated from moldy Chinese medicinal materials. It should be encouraged to use citral as mildew preventive agent for 
Chinese medicinal materials. Furthermore, the inhibition mechanism on $A$. flavus should be deeply clarified to provide more reliable theoretical basis.

\section{Data Availability}

All data and analyses are included as figures and tables within the manuscript.

\section{Conflicts of Interest}

The authors declare that they have no conflicts of interest.

\section{Authors' Contributions}

Guorong Fan contributed to this work.

\section{Acknowledgments}

The authors acknowledge the National Key Research and Development Program of China (2017YFD0600704) and Youth Talent Training Program of Jiangxi Academy of Forestry Sciences, China (2019522801), for financial support to carry out the work. They also thank Professor Dianming $\mathrm{Hu}$ and Bioengineering and Technological Research Centre for Edible and Medicinal Fungi (Jiangxi Agricultural University) for isolating and identifying $A$. flavus from moldy traditional Chinese medicinal materials.

\section{References}

[1] M. T. Hedayati, A. C. Pasqualotto, P. A. Warn, P. Bowyer, and D. W. Denning, "A. flavus: Aspergillus flavus: human pathogen, allergen and mycotoxin producer," Microbiology, vol. 153, no. 6, pp. 1677-1692, 2007.

[2] J. Yu, T. E. Cleveland, W. C. Nierman, and J. W. Bennett, "Aspergillus flavus genomics: gateway to human and animal health, food safety, and crop resistance to diseases," Revista Iberoamericana de Micología, vol. 22, no. 4, pp. 194-202, 2005.

[3] Z. Zhao, P. Guo, and E. Brand, "The formation of daodi medicinal materials," Journal of Ethnopharmacology, vol. 140, no. 3, pp. 476-481, 2012.

[4] S. Hu, X. Dou, L. Zhang, Y. Xie, S. Yang, and M. Yang, "Rapid detection of aflatoxin B1 in medicinal materials of radix and rhizome by gold immunochromatographic assay," Toxicon, vol. 150, pp. 144-150, 2018.

[5] I. Rizzo, E. Varsavsky, G. Vedoya, M. Haidukowski, H. Frade, and C. Chiale, "Fungal and aflatoxin contamination of medicinal herbs," Mycotoxin Research, vol. 14, no. 2, pp. 46-53, 1998.

[6] S. Amaike and N. P. Keller, "Aspergillus flavus," Annual Review of Phytopathology, vol. 49, no. 1, pp. 107-133, 2011.

[7] N. A. Nafady, S. A. M. Alamri, E. A. Hassan, M. Hashem, Y. S. Mostafa, and K. A. M. Abo-elyousr, "Application of $\mathrm{ZnO}$-nanoparticles to manage Rhizopus soft rot of sweet potato and prolong shelf-life," Folia Horticulturae, vol. 31, no. 2, pp. 319-329, 2019.

[8] P.-J. Chen, T. Moore, and S. Nesnow, "Cytotoxic effects of propiconazole and its metabolites in mouse and human hepatoma cells and primary mouse hepatocytes," Toxicology in Vitro, vol. 22, no. 6, pp. 1476-1483, 2008.

[9] M. Scordino, L. Sabatino, P. Traulo et al., "LC/MS/MS detection of fungicide guazatine residues for quality assessment of commercial citrus fruit," European Food Research and Technology, vol. 227, no. 5, pp. 1339-1347, 2008.

[10] J. Chen, Y. Shen, C. Chen, and C. Wan, "Inhibition of key citrus postharvest fungal strains by plant extracts in vitro and in vivo: a review," Plants, vol. 8, no. 2, p. 26, 2019.

[11] C. Chen, N. Cai, J. Chen, and C. Wan, "UHPLC-Q-TOF/MSbased metabolomics approach reveals the antifungal potential of pinocembroside against citrus green mold phytopathogen," Plants, vol. 9, no. 1, p. 17, 2020.

[12] B. A. Bahri, G. Mechichi, W. Rouissi, I. Ben Haj Jilani, and Z. Ghrabi-Gammar, "Effects of cold-storage facility characteristics on the virulence and sporulation of Penicillium expansum and the efficacy of essential oils against blue mold rot of apples," Folia Horticulturae, vol. 31, no. 2, pp. 301-317, 2019.

[13] V. Cardile, A. Russo, C. Formisano et al., "Essential oils of Salvia bracteata and Salvia rubifolia from Lebanon: chemical composition, antimicrobial activity and inhibitory effect on human melanoma cells," Journal of Ethnopharmacology, vol. 126, no. 2, pp. 265-272, 2009.

[14] N. Dikbas, R. Kotan, F. Dadasoglu, and F. Sahin, "Control of Aspergillus flavus with essential oil and methanol extract of Satureja hortensis," International Journal of Food Microbiology, vol. 124, no. 2, pp. 179-182, 2008.

[15] Y. Chen, Y. Wang, X. Han, L. Si, Q. Wu, and L. Lin, "Biology and chemistry of Litsea cubeba, a promising industrial tree in China," Journal of Essential Oil Research, vol. 25, no. 2, pp. 103-111, 2013.

[16] T.-T. Liu and T.-S. Yang, "Antimicrobial impact of the components of essential oil of Litsea cubeba from Taiwan and antimicrobial activity of the oil in food systems," International Journal of Food Microbiology, vol. 156, no. 1, pp. 68-75, 2012.

[17] H. Wang and Y. Liu, "Chemical composition and antibacterial activity of essential oils from different parts ofLitsea cubeba," Chemistry \& Biodiversity, vol. 7, no. 1, pp. 229-235, 2010.

[18] H. V. Nguyen, J.-C. Meile, M. Lebrun, D. Caruso, S. Chu-Ky, and S. Sarter, "Litsea cubeba leaf essential oil from Vietnam: chemical diversity and its impacts on antibacterial activity," Letters in Applied Microbiology, vol. 66, no. 3, pp. 207-214, 2018.

[19] E. Quintana-Rodriguez, L. E. Rivera-Macias, R. M. AdameAlvarez, J. M. Torres, and M. Heil, "Shared weapons in fungus-fungus and fungus-plant interactions? volatile organic compounds of plant or fungal origin exert direct antifungal activity in vitro," Fungal Ecology, vol. 33, pp. 115-121, 2018.

[20] J. Thielmann and P. Muranyi, "Review on the chemical composition of Litsea cubeba essential oils and the bioactivity of its major constituents citral and limonene," Journal of Essential Oil Research, vol. 35, no. 5, pp. 1-18, 2019.

[21] R. Kotan, S. Kordali, and A. Cakir, "Screening of antibacterial activities of twenty-one oxygenated monoterpenes," Zeitschrift Für Naturforschung C, vol. 62, no. 7-8, pp. 507-513, 2007.

[22] A. Ertas, M. Boga, M. A. Yilmaz et al., "A detailed study on the chemical and biological profiles of essential oil and methanol extract of Thymus nummularius (Anzer tea): rosmarinic acid," Industrial Crops and Products, vol. 67, pp. 336-345, 2015.

[23] S. B. Nerilo, G. H. O. Rocha, C. Tomoike et al., "Antifungal properties and inhibitory effects upon aflatoxin production by Zingiber officinale essential oil in Aspergillus flavus," International Journal of Food Science \& Technology, vol. 51, no. 2, pp. 286-292, 2015. 
[24] V. N. Trajano, E. D. O. Lima, and F. S. D. Souza, "Antifungal activity of the essential oil of Cinnamomum zeylanicum Blume and eugenol on Aspergillus flavus," Journal of Essential Oil Bearing Plants, vol. 15, no. 5, pp. 785-793, 2012.

[25] A. Kumar, R. Shukla, P. Singh, and N. K. Dubey, "Chemical composition, antifungal and antiaflatoxigenic activities of Ocimum sanctum $L$. essential oil and its safety assessment as plant based antimicrobial," Food and Chemical Toxicology, vol. 48, no. 2, pp. 539-543, 2010.

[26] A. Kumar, R. Shukla, P. Singh, C. S. Prasad, and N. K. Dubey, "Assessment of Thymus vulgaris L. essential oil as a safe botanical preservative against post harvest fungal infestation of food commodities," Innovative Food Science \& Emerging Technologies, vol. 9, no. 4, pp. 575-580, 2008. 